\title{
Context and Implications of Learning in Evolvable Production Systems
}

\author{
Pedro Neves João Ferreira Mauro Onori \\ KTH, Dep. of Production Engineering \\ neves@iip.kth.se, jfe@iip.kth.se, onori@iip.kth.se
}

\author{
José Barata \\ Universidade Nova de Lisboa \\ jab@uninova.pt
}

\begin{abstract}
More than ever the impact of market turbulence, high product customization and sustainability can be perceived through the increase of dynamics and complexity of manufacturing and business environments. Modular and distributed control structures are nowadays a consensual way, common to the majority of modern paradigms, to deal with unpredictability and volatility of markets. With modern paradigms aiming on adaptability, evolution and reconfiguration as a way to deal with this reality, learning mechanism have become very important to achieve such requirements. This paper presents an exploratory discussion on the topic of agent-based learning applied in a modern production paradigm such as Evolvable Production Systems (EPS).
\end{abstract}

\section{INTRODUCTION}

More than ever the impact of market turbulence, high product customization and sustainability can be perceived through the increase of dynamics and complexity of manufacturing and business environments. This is forcing major changes in industry and the traditional centralized and hierarchical approaches present themselves inadequate to face this new volatile reality. Fact that is widely accepted by main manufacturing roadmaps (e.g. Manufuture [1], Futman [2], etc) drawing the research line towards modularity and distributed characteristics of systems and architectures. Accordingly, in the last decades enormous research efforts have been conducted in order to introduce new manufacturing paradigms that can cope with this new market demands. Reconfigurable Manufacturing Systems (RMS) [3], Bionic Manufacturing Systems (BMS) [4], Holonic Manufacturing Systems (HMS) [5], Evolvable Assembly Systems (EAS) [6] and Evolvable Production Systems (EPS) [7] are some examples of modern paradigms that appeared to tackle this new challenges.

Modular and distributed control structures are nowadays a consensual way, common to the majority of modern paradigms, to deal with unpredictability and volatility of markets, forming a dynamic cooperative social network which results in distributed responsibilities, tasks, resources and behaviours that exceed the sum of the individual contributions. Agent architectures conceptualize an agent as an independent reactive/proactive entity, where each agent has perception, reasoning and evolvable capabilities. This type of architectures facilitates agent interactions under environmental constraints and allows the agent to take advantage of cooperation and social interactions. So, in this context the use of Multi-Agent Systems (MAS) is presented as the natural choice to implement modern paradigms. In [8, 9] two examples of implementations of such paradigms using MAS are presented.

EPS is a biologically inspired paradigm that relies on many simple, decoupled, intelligent and re-configurable modules or entities to support concepts such as adaptability and evolution. Evolution implies the re-configuration, removal or addition of physical modules in order to re-adjust the system to comply with new demands. On the other hand adaptability focuses on the re-adjustment of parameters, knowledge and functionality according to new product design and environment requirements. In this sense the adoption of learning behaviours is presented as a crucial feature giving the system the capacity to individually and collectively adapt itself, within their design purposes and limitations. The granularity and modularity characteristic of EPS foster the adoption of different learning behaviours at different levels of the architecture. Furthermore the interactions present at each level will also have a preponderant role inducing the selection between single agent leaning, where only the local knowledge is equated, and multi-agent learning, where the interactions and influence of the neighbours have also to be taken into account.

The purpose of this article is to discuss the development and exploitation of agent-based learning systems for dynamic networked Production Systems such as EPS.

The subsequent details are organized as follows: Section II gives an overview of agent-based Systems in Manufacturing; Section III frames the importance of learning as well as revise Learning methods for Agent-Based systems; Section IV contextualizes and underline potential benefits from Learning Systems for EPS; and finally Section V provide final remarks on the discussed subject.

\section{AGENT-BASED SYSTEMS IN MANUFACTURING}

The resulting pressure of the increase of complexity and customization of manufacturing environments have led to the inadequacy of classical centralized and hierarchical approaches applied production systems [10]. The need for novel manufacturing principles brought together the necessity of adopting new technologies. Agent technology provides a natural way to design and implement distributed production systems, due to agent core characteristics [11-16]. The most important features that support the use of agents in manufacturing are [17]: 
- Agents are autonomous, considering that they control their internal state and behaviours on the environment.

- Agents are rational since they can reason about the data they receive to better achieve their goal.

- Agents are adaptive in the sense they can learn and modify their behaviour according to the environment to better achieve their goals.

- Agents are goal oriented and proactive entities due to their capacity to have some control on their actions based on their internal goals

- Agents typically have incomplete information about environment

- Agents are social since they can communicate with other agents establishing a multi-agent system which can lead to collective and emergent behaviour

A MAS is composed by a network of agents that can interact and communicate with each other fostering the engagement of cooperation strategies. The distributed and social characteristics of the agents may allow the network to solve problems that are beyond the competences of the individuals. This may result in the resolution of otherwise untreatable problems. Moreover agents present features such as re-configurability, robustness, plug-ability, re-usability and learning which are key features to modern control units [18]. Due to the previously referred characteristics agent-based systems and technologies have a broad domain of application within production environment, providing promising solutions in presence of dynamic unpredictable environments where robust decision making and reconfiguration mechanisms are needed to deal with constant changes.

Nowadays there is a considerable number of implemented MAS control approaches to manufacturing that reinforce the suitability of MAS as a proper technology to support the modern manufacturing paradigms.

In [19] a product-resource-order-staff architecture (PROSA) which is a holonic reference for manufacturing systems uses three basic types of holons (product, order, resource) and a higher level holon (staff) to abstract products, resources, orders and logical activities. The resource holons are responsible for the control behaviour through an information processing part. The product holon holds the process and product knowledge. The order holon represent the tasks that can be executed by the system. Finally, the staff holon aims to assist the basic holons.

COBASA is a multi-agent based reference architecture that support fast adaptation and changes of shop floor control architecture with minimal effort [8]. In COBASA architecture aggregations of intelligent modules (coalitions) are orchestrated by coalition leaders in order to devise combined functionalities that match the product requirements.

ADACOR is an agile and adaptive manufacturing control architecture that increases agility and flexibility of enterprises, dealing with the need of fast reaction to disturbances at shop-floor level [9]. It introduces an adaptive control approach that evolves in time to achieve a combination between global production optimization and agile reaction to disturbances.
An implementation of a multi-agent production systems based on the EPS concept is presented in [20]. In this architecture each shop-floor module is abstracted by a generic mechatronic agent. Each mechatronic agent is composed by three main blocks such as the agent instantiator, the neighbourhood management and the skills orchestrator. The agent differentiation is only performed after the instantiation process where the agent embodies the module characteristics. This architecture was developed to support an emergent diagnostic system at the manufacturing network level.

Despite the number of implementation of multi-agent architectures based on modern manufacturing paradigms and the accomplishments already made, lot is yet to be achieved. Autonomous adaptation of modules is a complex and crucial aspect that has to be tackled in order to achieve real-time adaptation of production systems to new products demands. Moreover, evolution and optimization of processes to attain higher level of sustainability and competitiveness, capacity to deal and foster emergent behaviours as well as selforganization, are only some examples of the challenges that have not yet been completely solved and where learning can have a preponderant role.

\section{LEARNING IN AGENT-BASED SySTEMS}

Learning has been a research topic in a wide variety of domains. The increasing appeal and importance of agentbased systems have triggered the interest in both single and multi-agent learning systems. Machine Learning (ML) is one of the core fields of Artificial Intelligence (AI), which include features of learning in natural and technical systems. On the other hand, Distributed Artificial Intelligence (DAI) deals with learning in multi-agent systems. Despite the complementarity of these fields their intersection only started to be studied in the end of the 90's [21]. Even though ML has been vastly explored, its development did not take into account multi-agent environments. Hence the direct application of the ML algorithms to multi-agent architectures implies the abstraction of the interactions between agents. This means that in a straight forward application a ML mechanism can only work at agent level without taking into account the interactions with other entities and their influence. Any hint of multi-agent learning would have to be seen as an emergent property [22]. In a multi-agent learning approach systems are supposed to learn according to the domain where the agents are embedded into. Even if an agent is unaware of the presence of others their influence in the environment is reflected in the learned behaviour and knowledge.

Independently of the used architecture, learning approaches can be divided into three main groups: supervised, unsupervised and reward-based learning. The differentiation aspect is the type of the feedback that the learner system receives.

Despite the growing efforts that have been put in the development of agent-based learning systems last decade, only a relatively small amount is related with agent-based 
manufacturing learning systems. Therefore a brief review of the typical methods used in agent-based learning will be presented covering some other application domains in addition to manufacturing agent-based learning systems. Moreover the further review will be focused in multi-agent learning methods since for the implementation of single-agent learning classical and already thoroughly studied methods can be used. Notwithstanding this methods are as crucial as multiagent learning methods to EPS and similar production systems.

A considerable number of multi-agent learning applications are focused on reinforcement learning. Although reinforcement learning was not developed for multi-agent environments, some developed approaches were thought to counter the problems faced by such systems. Three case studies of multi-agent reinforcement learning involving cooperation by sharing sensations, learned policies and episodes are presented in [23]. Through the study cases was possible to conclude that through cooperation and exchanging information, agents can learn faster and converge sooner than independent agents. However, if extra information is not prudently used it can interfere with learning. Sharing knowledge or episodes to achieve cooperation comes also with a communication cost which, if not deal properly can have adverse effects. Therefore in a multi-agent system a balance between exchanged information and communication cost must be achieved.

In [24] an algorithm for distributed reinforcement learning based on Q-learning is presented. A projection that can be interpreted as a kind of 'optimistic assumption', where each agent works as if its teammates complete its behaviour to achieve an optimal behaviour was introduced. Furthermore, an additional procedure for cooperation in-between the team was developed. In the end was established that to achieve distributed reinforcement learning additional coordination techniques are necessary. Kapetanakis and Kudenko introduced in [25] an approach that aims to achieve the convergence of the agents to the optimal joint action. To achieve such goals two types of learners were used: an independent and a joint-action learner. The independent leaner is unaware of the existence of other agents whereas the joint-action can also perceive the action of the others. Similarly to the previous articles the author used the QLearning algorithm for the learning technique. However, in a single-agent scenario Q-Learning ensures the convergence to the optimal action independently of the action selection strategy, in a multi-agent setting, the action selection strategy becomes crucial for convergence to any joint action. The designated selection strategy was Boltzmann strategy [26]. Some of the achieved conclusions was that joint action can be attained not only ensuring that the temperature function adheres to certain properties but also choosing the proper estimated value function.

An analysis of the importance of rewards in multi-agent reinforcement learning is made in [27].The intervention of more than one learning entity creates a dynamic environment which leads to the necessity of the agents to based their decision on a history of joint past actions and on how they would like to influence future ones. The agents implement the Q-Learning algorithm with the objective of learn a policy that maximizes their long-term rewards. The only way to achieve long-term reward is through cooperation. The conclusion was that Q-learners are able to behave efficiently in multi-agent settings when the reward function is evolved.

A model for cooperative learning based on structured dialog between agents is presented by Sian in [28]. In the presented model each agent starts by learning locally. When an agent formulates a hypothesis in which it has reasonable confidence it proposes it to the other agents via an interaction board. Through the previously gained experience the other agents will evaluate the hypothesis that is accepted if approved by consensus. The authors concluded that multiagent system performed the inductive generalization faster than the single-agent systems. Furthermore, in the presence of a great amount of data a multi-agent system will give advantages in terms of speed.

Another different approach is collective memory based multi-agent learning system presented by Garland and Alterman in [29]. Collective memory (CM) is the resources that the agents gain through experience and use to improve their performance when interacting to solve collaborative problems. The agents maintain both a procedural knowledge $\mathrm{CM}$ and also an operator probabilities CM. It is author's belief that mechanism of collective memory lead to efficient long-term behaviour even if short-term behaviour is suboptimal. The results showed that $\mathrm{CM}$ is a mechanism through which a community of agents can learn improved patterns of cooperative activity taking advantages of regularities in their domain of problem-solving activity.

Since we live in a world that is populated by human beings, intelligent social entities that are dependent on the success of cooperation between peers it is logic to take advantage of the knowledge that humans possess to improve the performance of a system. Therefore, and since we can consider a human being as an intervenient agent, we will present two articles were the interaction between agent and human is taken into account.

In [30] a social agent that aims to interact individually with each user facilitating personalized information and taking meaningful actions to the users is presented. The agent communicates through rudimentary chat abilities. The learning mechanism is base in a system of rewards and punishments (reinforcement learning). Due to the dynamicity of the environment and the characteristics of the human being the application has to deal with a number of challenges such as multiple reward sources, inconsistency of user rewards and desires, data sparsity, among others. The results were found compelling and proved promising in the application of reinforcement learning in rather rich and open-ended social settings. In [31] a collaborative task learning agent is presented. The purpose of the article is demonstrate that it is possible to achieve good results learning executable task 
models from a single collaborative learning session consisting of demonstration, explanation and dialogue. The core challenge is identifying the appropriate generalization, which are the key to allow the learned task model to be successfully applied to new arguments in new contexts. The agent learns combining information about what the user says and which actions the user performs. Through a heuristic search process of combine a structural distance metric and a semantic metric the agent attempts to produce generalist conceptual rules.

Regarding the application of agent-based systems in the manufacture some articles will be reviewed to demonstrate possible applications of the leaning behaviour. Shen, Maturana et al. [32] present two learning mechanisms: learning from history and learning from the future through forecasting. The learning from history mechanism is based on distributed case-based learning approach for capturing behavioural patterns at the resource mediator level to further store as knowledge. The learning from future through forecasting uses a simulation system as a base to propagate the system's behaviour into a near future and acquire an estimated heuristic value that can be used to update the system knowledge. Through the obtained results was concluded that the mechanisms are useful to enhance coordination capabilities and performance of agent-based manufacturing system. The learning from the future mechanism can also provide important information to adjust the performance of the system. In [33] a learning mechanism is presented to adapt the diagnostic system of an EPS to the failure history of the agent. The Baum-Welch algorithm explores the third problem of the Hidden Markov Models to implement the learning mechanism.

A review on the application of a number of learning techniques to deal with changes and uncertainties in manufacturing systems is presented in [34].

Multi-agent learning denotes a number of challenges raised by the characteristics of these systems. The distribution of the knowledge between modules can lead to a completion problem, through which the agents have to learn with incomplete data. The impossibility of validate an hypothesis with a secure level of confidence, due to the incompleteness of information [28] is the cause for the confidence problem. This problem is a huge obstacle to the adoption of unsupervised learning by production systems, since it has to be ensured that the agent or the agents network learn the correct knowledge otherwise the consequences on the system behaviour might be catastrophic. The Scalability is also a challenge for learning techniques applied to MAS, since the dimensionality of the search space grows rapidly with the number and complexity of agent behaviours and the number of actors. The complexity generated by the interaction between agents can also cloud the sensed environment by the agent, deteriorating the learning behaviour [35]. Multi-agent systems are typically dynamic systems which present a unique challenge not usually found in single-agent learning: as the agent learns, the adaptation of the agents to one another changes the world scenario.
Despite the challenges that need to be tackled, multi-agent learning behaviours promise to have a very important role in distributed production systems, since they provide among others faster learning capabilities and more capacity to deal with great amount of data.

\section{CONTEXT AND IMPLICATION OF LEARNING IN EPS}

\section{A. Contextualization}

As a modern production system EPS envisions evolution and adaptation. To support such concept EPS relies in fine granularity at the shop floor level, where each component is a skill-oriented mechatronic agent. A mechatronic agent (MA) is a software construct that embodies specific equipment and maps its functionalities into EPS interaction dynamics. Moreover, in this context a skill is a set of attributes that model the characteristics and control actions that represent the action which is performed whenever the skill is executed. In EPS environment the skills are classified in basic (atomic) skills and complex (composed) skills. The basic skills are the processes inherent to the module design. Complex skills are skills that result from the self-organization and cooperation between modules and coalitions of modules. However, in a more theoretical approach complex skills can emerge from the unexpected conjugation and interaction between modules.

The modular and pluggable approach followed in EPS foster the dynamic formation of modules coalitions as a result of multi-agent negotiation and composition rules. Whenever such dynamic coalitions are established, a coalition leader takes action to represent the coalition as higher level process (complex skills) present in the system (Fig. 1). The product requirements are then derived from product design and matched against the available skills in the system. In this sense if new product requiring new processes is introduced into production, the system has to evolve structurally. Therefore in EPS environment evolution is more a long term property rather than adaptability which has to be run-time to overcome disturbances such as equipment failures, changes in production and product requirements, among others.

Adaptability and evolution have repercussions that impose changes at different system levels. For that reason a three level model was established to classify the changes

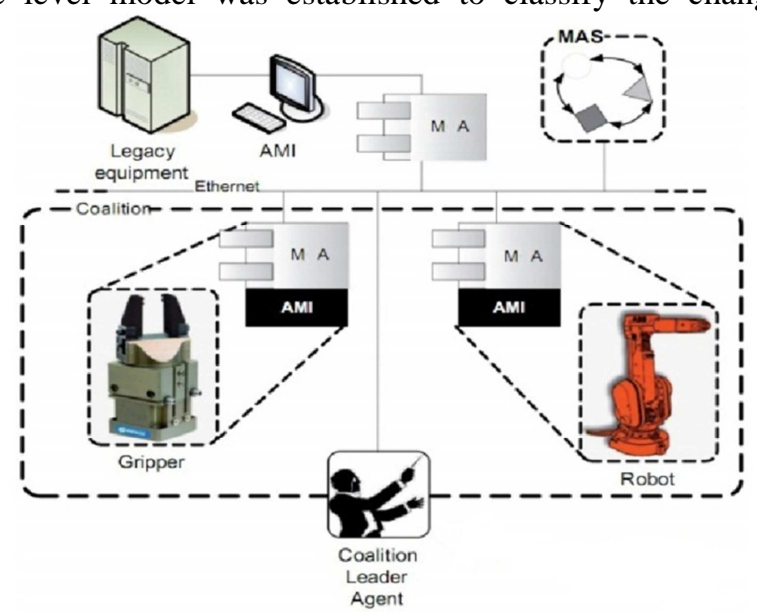

Fig. 1. Multi-agent basic architecture. Adapted from [38]. 
according to the involved system levels [36]:

- Level 0: Parametric Changes - adapting the behaviour of available capabilities; e.g. changing the force settings of a pressing device.

- Level 1: Logical Changes - adapting the utilization of available capabilities (skills); e.g. change of process sequences from one product to another.

- Level 2: Structural Changes - adapting the available capabilities; e.g. changing one process module for another one or adding/removing modules

Supporting such dynamic and self-capable architecture means that EPS highly depend on learning capacities to fully reach its potential. In this sense and according to EPS modularization, learning behaviours can be explored at three levels (Fig. 2). The first level is the module level, which represent the basic building block of the EPS System. The second level is the coalition level, which encompasses two or more modules/coalitions that together provide at least one complex skill. Finally, the global level which respect the totality of the system (MAS) in which cooperation leads to the realization of the product workflow.

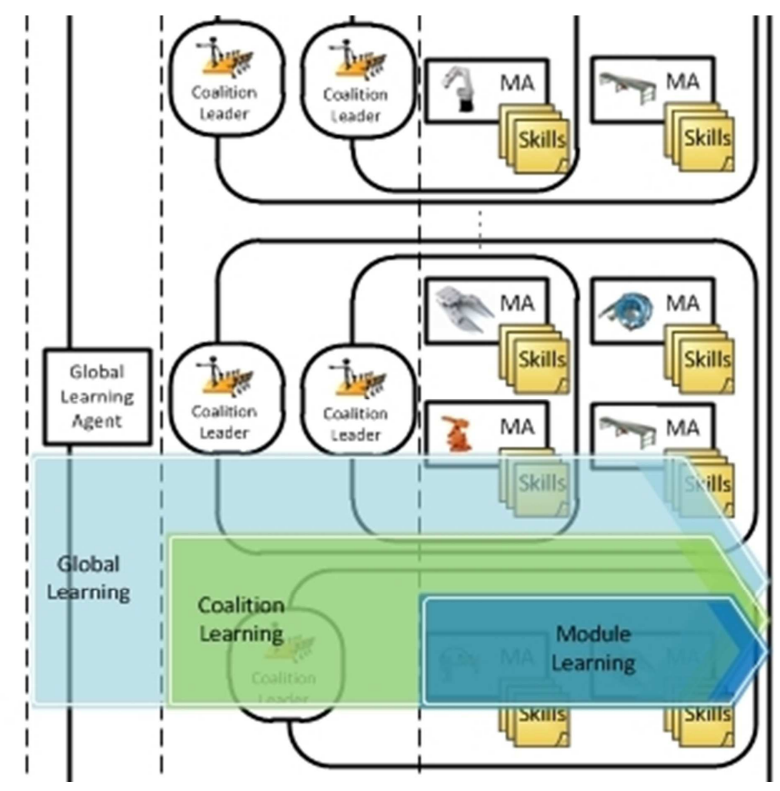

Fig. 2.Learning levels in EPS.

\section{B. Learning implications}

At the module level, learning can have a major role in parameter optimization enabling module flexibility. In this context module flexibility imply the capacity of the module to adjust its parameterization to better match the product requirements. For instance, if we think the module as a feeder station we can foresee a learning behaviour where the module can adjust and learn the feeding rate through a feedback system and achieve the final goals in a more efficient way. At this level the learning is presented as a case of single learning. For the module parameter optimization a classical learning algorithm could be used adjusting the values in real time in order to optimize the module performance. A very important aspect that cannot be forgotten is the knowledge of the system that human experts usually possess, which is normally ignored and that should be used as input of the learning system to improve the modules performance.

Whenever new modules are deployed into the system new interactions are established and new skills self-organize, providing the system new complex processes (complex skill). Simultaneously a generation of new coalitions takes place. Each coalition is represented by a coalition leader agent that is responsible for the managing of the complex skills as well as for the coalition members. At this point it is important to reinforce that a coalition member can be part of another coalition. At the coalition level, a multi-agent learning behaviour should account the effect that changes in the module parameterization cause in the adjacent modules and coalitions. This way decisions could be made regarding what modules parameters should be optimized aiming the improvement of the overall performance. The autonomous management of modules and skills, also requires learning capabilities, due to the dynamic characteristic of the system. More specifically a learning behaviour which would be able to capture the logic relations that enable the conjugation of skills would foster the self-organization and provide the system the autonomy to deal with the emergence especially in cases of high complexity systems. Possible approaches would involve grammar capture and movement description languages. At a structural level the addition of certain modules may lead to the establishment of successful profitable coalitions. In this sense, information about the successfulness plug ability of new modules can be taken as an important asset to establish future coalitions that are known from the start that will originate successful efficient processes. Since coalitions are dynamic the stored information need to be kept. The knowledge could be inherited by the involved modules. If similar modules are further added into the system the knowledge could also flow between peers to improve the module performance from history based knowledge.

Finally at the global level real-time information about performance of cooperation between coalitions as well as information about overall system performance could be learned and used to improve the accomplishment of the workflow. A global agent that gathers information about the system performance and that can affect the newly instantiated product agents in real time could be beneficial for the system to tackle unpredictable situations. This agent would be responsible to collect information about the other agents and suggest the best workflow given the current status of the system. Other goal for a global learning agent would be the exploitation of learned historical data (long-term information). New optimized design configurations could be deduced and used in future evolutions of the same system or the design of entirely new systems. The global learning agent targets global optimization and therefore is closely related to the product agents and the successful accomplishment of their workflow since this represents the global system objective. 


\section{FINAL REMARKS}

This paper presented an exploratory discussion on the topic of learning for EPS and the fundamental requirements and implications. As it was presented modern learning systems play a crucial role in modern and future production paradigms.

Future research will be conducted to investigate the methods and approaches that are more suitable for each level of Learning in EPS. Nevertheless lot of work also as to be developed to overcame the presented challenges inherent to such dynamic adaptable distributed systems.

\section{REFERENCES}

[1] F. Jovane, et al., The ManuFuture Road: Towards Competitive and Sustainable High-Adding-Value Manufacturing: Springer Verlag, 2008.

[2] FutMan, "The Future of Manufacturing in Europe 2015-2020 the Challenge for Sustainability, Institute for Prospective Technological Studies," European Commission Joint Research Centre, 2006.

[3] H. A. ElMaraghy, "Flexible and reconfigurable manufacturing systems paradigms," International Journal of Flexible Manufacturing Systems, vol. 17, pp. 261-276, 2005.

[4] K. Ueda, "A concept for bionic manufacturing systems based on DNA-type information," 1992, pp. 853-863.

[5] R. F. Babiceanu and F. F. Chen, "Development and applications of holonic manufacturing systems: a survey," Journal of Intelligent Manufacturing, vol. 17, pp. 111-131, 2006.

[6] M. Onori, et al., "Evolvable Assembly Systems Basic Principles," 2006, p. 317.

[7] J. Barata, et al., "Evolvable Production Systems: Context and Implications," 2007.

[8] J. Barata, "Coalition based approach for shopfloor agility," 2005.

[9] P. Leitão and F. Restivo, "ADACOR: a holonic architecture for agile and adaptive manufacturing control," Computers in Industry, vol. 57, pp. 121-130, 2006.

[10] V. Marik and D. McFarlane, "Industrial adoption of agent-based technologies," IEEE Intelligent Systems, pp. 27-35, 2005.

[11] H. Park, et al., "Agile infrastructure for manufacturing systems (AIMS): A vision for transforming the us manufacturing base," 1993.

[12] A. Kwok and D. H. Norrie, "A development system for intelligent agent manufacturing software," Integrated Manufacturing Systems, vol. 5, pp. 64-76, 1994.

[13] M. R. Cutkosky, et al., "Madefast: collaborative engineering over the Internet," Communications of the ACM, vol. 39, 1996.

[14] F. P. Maturana and D. H. Norrie, "Multi-agent mediator architecture for distributed manufacturing," Journal of Intelligent Manufacturing, vol. 7, pp. 257-270, 1996.

[15] V. Parunak, et al., "AARIA agent architecture: an example of requirements-driven agent-based system design," Citeseer, 1997.

[16] W. Shen, et al., "An agent-based manufacturing enterprise infrastructure for distributed integrated intelligent manufacturing systems," 1998, p. 550.

\section{CIRP Annals-Manufacturing Technology, vol. 55, 2006}

P. Leitão, "Agent-based distributed manufacturing control: A state-of-the-art survey," Engineering Applications of Artificial Intelligence, vol. 22, pp. 979-991, 2009.

[19] H. Van Brussel, et al., "Reference architecture for holonic manufacturing systems: PROSA," Computers in industry, vol. 37, pp. 255-274, 1998.

[20] J. B. a. J. F. Luis Ribeiro, "An Agent-Based Interaction-Oriented Shop Floor to Support Emergent Diagnosis," 8th IEEE International Conference on Industrial Informatics, Japan, 2010.

G. Weiss, Multiagent systems: a modern approach to distributed artificial intelligence: The MIT press, 1999.

E. Alonso, et al., "Learning in multi-agent systems," The Knowledge Engineering Review, vol. 16, pp. 277-284, 2001.

M. Tan, "Multi-agent reinforcement learning: Independent vs. cooperative agents," 1993.

M. Lauer and M. A. Riedmiller, "An algorithm for distributed reinforcement learning in cooperative multi-agent systems," 2000, pp. 535-542.

[25] S. Kapetanakis and D. Kudenko, "Reinforcement learning of coordination in cooperative multi-agent systems," 2002

[26] L. P. Kaelbling, et al., "Reinforcement learning: A survey," Arxiv preprint cs/9605103, 1996.

[27] V. Vassiliades and C. Christodoulou, "Multiagent Reinforcement Learning in the Iterated Prisoner's Dilemma: Fast cooperation through evolved payoffs," 2010, pp. 1-8.

[28] S. Sian, "Extending learning to multiple agents: issues and a model for multi-agent machine learning (MA-ML)," 1991.

[29] A. Garland and R. Alterman, "Multiagent learning through collective memory," 1996, pp. 33-38.

[30] C. Isbell, et al., "A social reinforcement learning agent," 2001, pp. 377-384.

[31] J. Allen, et al., "Plow: A collaborative task learning agent," 2007, p. 1514.

[32] W. Shen, et al., "Enhancing the performance of an agent-based manufacturing system through learning and forecasting," Journal of Intelligent Manufacturing, vol. 11, pp. 365-380, 2000.

[33] J. B. a. J. F. Luis Ribeiro, "A co-Evolving Diagnostic Algorithm for Evolvable Production Systems: A Case of Learning," 2010.

[34] L. Monostori, "AI and machine learning techniques for managing complexity, changes and uncertainties in manufacturing," Engineering Applications of Artificial Intelligence, vol. 16, pp. 277-291, 2003.

[35] D. A. Levinthal and J. G. March, "The myopia of learning," Strategic management journal, vol. 14, pp. 95-95, 1993.

[36] N. Lohse, "Towards an ontology framework for the integrated design of modular assembly systems," 2006.

[37] A. Maffei and M. Onori, "Evolvable Production Systems: Environment for New Business Models," Key Engineering Materials, vol. 467, pp. 1592-1597, 2011.

[38] G. Cândido and J. Barata, "A Multiagent Control System for Shop Floor Assembly," Holonic and Multi-Agent Systems for Manufacturing, pp. 293-302, 2007. 